so far. Due to retrospective data we choose as outcome only treatment duration and adverse events, direct outcomes of efficacy were impossible to evaluate. Our results indicates a poor treatment duration of biologics given off label in CTDs with a relevant prevalence of adverse events and failures. It has to be underlined that our population was mainly on TNF blockers.

These data discourage the use of biologics, mainly of TNF blockers in CTDs, even if they still can be considered with caution in very selected cases after failure of the other on label medications.

Disclosure of Interest: None declared

DOI: 10.1136/annrheumdis-2017-eular.2952

\section{AB0450 THE DARK SIDE OF GLUCOCORTICOID THERAPY IN SYSTEMIC LUPUS ERYTHEMATOSUS: CAN WE DO SOMETHING ABOUT THAT?}

I. Saulescu, T. Gudu, A. Neagu, D. Opris, A. Borangiu, S. Daia-lliescu, L. Groseanu, A. Balanescu, V. Bojinca, R. Ionescu. Internal Medicine and Rheumatology, Umf Carol Davila, Sf. Maria Hospital, Bucharest, Bucharest, Romania

Background: Corticosteroids are still one of the main treatment in Systemic Lupus Erythematosus (SLE). Beside the effect on controlling disease activity, they are also implicated in damage accrual. Both patients and physicians are some time afraid to adopt a steroid free regimen when possible.

Objectives: To evaluate the knowledge and perception of patients with SLE upon glucocorticoids.

Methods: 84 patients with SLE were evaluated and data about demographic, clinical, serological characteristics or treatment were collected. Presence of steroids related side effects like hypertension, osteoporosis, cataracts or diabetes mellitus were also assessed. All patients completed a questionnaire in order to evaluate patient's knowledge about steroids. They were asked if they had a discussion with the doctors about corticotherapy and side effects related to them, if they consider that this treatment could be stopped with specialist approval. Statistics was performed with SPSS program.

Results: All patients had treatment with corticosteroids during disease evolution. $57.14 \%$ of them experienced at least one steroids related side effect. This patients were significant older: mean age at evaluation 49.50 versus $36.47(p<0.0001)$, had a longer disease duration: mean SLE duration 9.27 versus 4.69 (p0.016), a higher mean Prednisone equivalent dose: 8.86 versus 4.71 ( $p$ 0.031), a higher mean SLICC Damage Index: 1.53 versus 0.44 ( $p$ 0.001) than patients without steroids related side effects. This complications were significantly more rare in patients that were on a steroid free regimen at the moment of evaluation versus those on a continuum steroid regimen $(7.14 \%$ versus $50 \%, p<0.0001)$.

When patients were asked if they will stop steroids according to medical advice, almost $1 / 3$ of patients $-28.57 \%$ - responded "no- to afraid to do that". Patients willingness to adhere to a steroid free regimen in the future according to a physician recommendation was significant more frequent in younger patients $(p$ $0.031, r-0.235)$, in those with steroids initiated in less than 1 year $(p 0.016, r$ -0.297 ) and in those with less damage accrual ( $p 0.017, r-0.267)$. Flare at the moment of evaluation significantly reduced this possibility, at least from the patient perspective ( $\mathrm{p} 0.041, \mathrm{r} 0.224)$. The likelihood of a future steroid free regimen was increased by a previous discussion patient-doctor about steroids ( $\mathrm{p} 0.002$ ).

Conclusions: This study clearly shows that an open discussion with our SLE patients about corticosteroids is mandatory from the beginning. Patients should be informed about possibility of a steroid free regimen when disease status permits. This will increase patient willingness to get free of steroids when possible, helping physician to limit the continuum damage accrual of SLE patients.

References:

[1] Duru N, van der Goes MC, Jacobs JWG et al, " EULAR evidence-based and consensus-based recommendations on the management of medium to high-dose glucocorticoid therapy in rheumatic diseases", Ann Rheum Dis 2013; 72: 1905-1913.

Disclosure of Interest: None declared

DOI: 10.1136/annrheumdis-2017-eular.3978

\section{AB0451 RIVAROXABAN VERSUS WARFARIN AS SECONDARY THROMBOPROPHYLAXIS IN PATIENTS WITH ANTIPHOSPHOLIPID SYNDROME PROTOCOL: A RANDOMIZED, MULTICENTRE, OPEN-LABEL, CLINICAL TRIAL}

J. Cortés-Hernández ${ }^{1}$, L. Saez-Comet ${ }^{2}$, M. Perez-Conesa ${ }^{2}$, A. Riera Mestre $^{3}$, A. Castro-Salomo ${ }^{4}$, S. Parra ${ }^{4}$, J. Cuquet-Pedragosa ${ }^{5}$, V. Ortiz-Santamaria ${ }^{6}$, M. Mauri-Plana ${ }^{7}$, P. Suñe ${ }^{8}$, J. Ordi-Ros ${ }^{9} .{ }^{1}$ Vall Hebron Institute Research (Vhir), Barcelona; ${ }^{2}$ Autoimmune Unit. Internal Medicine Department, Miguel Servet Hospital, Zaragoza; ${ }^{3}$ VTE Unit. Internal Medicine Department, Bellvitge University Hospital, Barcelona; ${ }^{4}$ Internal Medicine Department, Sant Joan de Reus University Hospital, Reus; ${ }^{5}$ Internal Medicine Department; ${ }^{6}$ Rheumatology Unit, Granollers University Hospital, Granollers; ${ }^{7}$ Internal Medicine Department., Mataró Hospital, Mataró; ${ }^{8}$ Pharmacy Department, Vall d'Hebron Hospital;

${ }^{9}$ Autoimmune Unit. Internal Medicine Department, Vall Hebron Institute Research (Vhir), Barcelona, Spain

Background: Long-term anticoagulation is widely used for secondary throm- boprophylaxis in the antiphospholipid syndrome (APS) due to the high risk of recurrent events. Currently anticoagulation with vitamin $\mathrm{K}$ antagonists (VKAs) is the standard of care but have unpredictable pharmacodynamic properties that requiere monitoring for dose adjustment. Rivaroxaban, an orally active direct factor Xa inhibitor, has been shown to be effective and safe compared with warfarin for the treatment of venous thromboembolism and non valvular atrial fibrillation in major RCTs. No studies had been published in APS.

Objectives: To investigate the efficacy and safety of rivaroxaban in preventing recurrent thrombosis in patients with APS compared with warfarin.

Methods: This is a phase 3 randomized, multicenter, non-inferiority open-label RCT. 190 eligible APS patients with arterial or venous thrombotic history receiving warfarin will be stratified according the presence of SLE and venous/arterial thrombotic history and randomized (1:1) either to continue warfarin (standard of care, normalized ratio (INR) 2-3 or 2.5 to 3.5 in those with recurrent thrombotic episodes) or to switch to rivaroxaban $(20 \mathrm{mg} /$ day). The primary efficacy outcome is the development of any thrombotic event during the study period. Secondary efficacy outcomes include time to thrombosis, type of thrombosis (arterial or venous), overall causes of death, evaluation of a prognostic biomarker panel of recurrent thrombosis. The primary safety outcome will be major bleeding. Secondary safety outcomes include any adverse event and minor bleeding. The study has 3 years follow-up. First patient was included in March 2013 (EUDRA-CT:2010-019764-36).

Conclusions: If the study demonstrates a non-inferior anticoagulant effect compared with warfarin, this would provide sufficient supporting evidence to make rivaroxaban a standard of care for the treatment of patients with APS with previous thrombotic history.

Disclosure of Interest: None declared

DOI: 10.1136/annrheumdis-2017-eular.6286

\section{AB0452 CASE REPORT OF ALLOGENEIC UNRELATED-DONOR MESENCHYMAL STEM CELLS (MSC) INFUSION IN SJOGREN SYNDROME (SS) WITH REFRACTORY THROMBOCYTOPENIA}

J. Qi, M. Qu, J. Gu. Department of Rheumatology, Third Affiliated Hospital of Sun Yat-sen University, Guangzhou, China

Background: Intravenous MSC infusion has been reported occasionally in SS treatmen and Immune Thrombocytopenia ttreament. To our knowledge,there is rare report of allogeneic unrelated-donor intravenous MSC infusion in SS with thrombocytopenia without immunosuppresive induction of MSC transplantation.

Objectives: To report the case of a Sjogren syndrome with refractory thrombocytopenia treated with MSC infusion. To review the current literature on intravenous MSC infusion for SS.

Methods: Literature review and multidisciplinary discussion were thoroughly performed before treatment protocol was approved by institutional ethics committee and patient and family signed informed consent form.

Results: A 48 year-old female with a 2-year history of SS, manifested by severe thrombocytopenia (PLT 14-30*10E9/L, after each injection of recombinant human interleukin 11 , platelets can be transiently recovered.) refractory to Methylprednisolone, methotrexate, azathioprine and cyclophosphamide received Four infusions (Once a month) of allogeneic unrelated-donor $2 \times 10^{6} / \mathrm{Kg} \mathrm{MSC}$. After two infusiong of MSC, the PLT increased gradually to greater than $100^{*} 10 \mathrm{E} 9 / \mathrm{L}$. After 1 year of follow-up, Platelet counts remained normal.

Conclusions: These results suggest that mesenchymal stem cells may be a therapeutic strategy for Sjogren syndrome with Refractory thrombocytopenia patients. Larger studies are needed to validate clinical efficacy and safety and to standardize treatment protocol of MSC infusion in SS.

References:

[1] Zhang D, Li H, Ma L, et al. The defective bone marrow-derived mesenchymal stem cells in patients with chronic immune thrombocytopenia. Autoimmunity 2014, 47 (8), 519-29.

[2] Xu J, Wang D, Liu D, et al. Allogeneic mesenchymal stem cell treatment alleviates experimental and clinical Sjogren syndrome. Blood 2012, 120 (15), 3142-51.

[3] Wu M, Ge H, Li S, et al. Mesenchymal Stem Cells Immunosuppressed IL-22 in Patients with Immune Thrombocytopenia via Soluble Cellular Factors. Journal of immunology research 2015, 2015, 316351.

Disclosure of Interest: None declared

DOI: 10.1136/annrheumdis-2017-eular.6643

\section{AB0453 OFF-LABEL USE OF MYCOPHENOLATE IN PATIENTS WITH SYSTEMIC LUPUS ERYTHEMATOSUS IN A RHEUMATOLOGY CENTER IN COLOMBIA}

${\mathrm{K} . \text { Mendez }^{1}}^{1}$, D. Buitrago-Garcia ${ }^{2}$, P. Santos-Moreno ${ }^{3}$, J. Urrego ${ }^{4}$

${ }^{1}$ Pharmaceutical chemist; ${ }^{2}$ Epidemiology; ${ }^{3}$ Rheumatology, Biomab, Center for

Rheumatoid Arthritis, Bogota; ${ }^{4}$ Pharmaceutical chemist, UDCA University,

Bogota, Colombia

Background: Immunosuppressants such as mycophenolate are widely used in people with systemic lupus erythematosus (SLE), but not all are specifically licensed by FDA, EMA (1) and in the case of Colombia the Regulatory Agency for Food and Drugs (INVIMA) has not approved it for this indication, only for the 